\title{
Afiliación intelectual y escritura en la universidad: Un estudio de caso sobre la perspectiva de los docentes
}

\author{
Miriam Casco \\ Universidad Nacional del Centro de la Provincia de Buenos Aires, Argentina
}

Artículo recibido 17 de enero de 2012; aceptado 15 de febrero de 2013; versión final recibida 30 de marzo de 2014

\begin{abstract}
El presente trabajo constituye un ejercicio de aproximación a la perspectiva del profesorado frente a los problemas intelectuales y discursivos de los estudiantes universitarios. Los datos que se analizan fueron obtenidos mediante la administración de una encuesta y la realización de entrevistas a docentes de tres áreas de formación (Ciencias Sociales, Ciencias Veterinarias e Ingeniería). El trabajo de campo se realizó en la Universidad Nacional del Centro de la Provincia de Buenos Aires, Argentina. En la exposición de resultados se identifican preconcepciones y prácticas docentes con el fin de evaluar su posible gravitación en los procesos de incorporación a la cultura universitaria.
\end{abstract}

\section{Introducción}

El tema de la lectura y la escritura en la educación superior comenzó a hacerse un lugar en los programas de investigación de las universidades argentinas a mediados de los años ' 80 y cobró un impulso notable en las siguientes décadas. La constitución de este nuevo campo de indagaciones coincidió con una histórica expansión de la matrícula; es decir que el estudio de las prácticas comunicativas en el medio universitario nació ligado al fenómeno de la masificación y, con ello, a dos de sus consecuencias negativas: el rezago y la deserción.

En virtud de la generalizada percepción de que se estaban operando notables cambios en el rendimiento comunicativo de las nuevas generaciones, y frente a la necesidad de dotar de fundamento científico a los dispositivos de intervención pedagógica, durante los años siguientes se produjeron investigaciones basadas en tres disciplinas de referencia: la lingüística, la psicología y las ciencias de la educación (Carlino, 2007). En su mayoría se trata de estudios diagnósticos centrados en los déficits comunicativos de los estudiantes universitarios: los 'nuevos estudiantes' ya no leen ni escriben conforme lo esperado por la institución universitaria y es preciso explicar las razones de tal fenómeno.

$\mathrm{La}$ inconveniencia de centrar las indagaciones en las carencias de los estudiantes ha sido tempranamente reconocida por los investigadores en diversos espacios de debate 
e intercambio. Sin embargo, siguen siendo minoritarios los trabajos que buscan entender el fracaso tomando en consideración, entre otros factores, el contexto institucional, las tradiciones académicas según áreas de estudio, o el rol de los actores institucionales en el proceso de inserción. Precisamente en relación con este último aspecto, se hace necesario examinar mucho más la perspectiva de los docentes a fin de evaluar la gravitación de sus preconcepciones y de sus prácticas en el desempeño estudiantil. El estudio que se presenta a continuación ofrece un acercamiento a esta cuestión a partir de un trabajo de campo realizado en la Universidad Nacional del Centro de la Provincia de Buenos Aires, Argentina

Sabemos que el ingreso a la universidad constituye un proceso complejo en el que interactúan múltiples factores contextuales y personales (Aguilar Rivera, 2007; Araujo, 2008). Por ello nos preguntamos: ¿cuáles son las percepciones, creencias, representaciones de los docentes frente a ese proceso? y ¿cuál es el papel que le otorgan a las prácticas de escritura en el mismo? Con el propósito de recoger información al respecto hemos realizado un ejercicio de aproximación al tema mediante la administración de una encuesta y la realización de entrevistas a profesores universitarios.

\section{Fundamentos teóricos}

La llegada de un individuo a la universidad no implica la progresión natural de un nivel educativo a otro; por el contrario, puede considerarse un complejo proceso de entrada a otra cultura. Esta es la perspectiva sostenida por un conjunto de investigaciones provenientes de la sociología francesa de los años '90 (Guzmán Gómez, 2002). En el marco de esa corriente teórica Coulon $(1995,1997)$ estableció las bases conceptuales para pensar el ingreso a la universidad. A partir de supuestos debidos a la sociología, el interaccionismo y la etnometodología de la educación, explica la entrada a la universidad como tránsito o pasaje de un estatus social a otro, de una cultura a otra. Se trata, según el autor, de un proceso en tres tiempos: el tiempo de la alienación (el recién llegado es extranjero en un ámbito que rompe con su mundo anterior); el tiempo del aprendizaje (el ingresante moviliza energías, define estrategias, se adapta progresivamente); y el tiempo de la afiliación (el individuo puede demostrar un relativo dominio de las normas). 
Para ser admitido en la nueva cultura el ingresante debe aprender su oficio de estudiante, lo cual implica una iniciación a las reglas de la universidad en dos planos, el institucional y el intelectual. En este segundo plano el éxito del recién llegado depende del descubrimiento, comprensión y manejo de los códigos del trabajo intelectual. Para lograr la afiliación intelectual el nuevo estudiante debe abrirse paso en un terreno de conceptos, categorizaciones, discursos y prácticas privativos de la educación universitaria (Coulon, 1995; 1997).

La afiliación se produce cuando los recién llegados logran establecer una consonancia parcial con la cultura universitaria y han emprendido la construcción de una nueva identidad. Ese logro entraña un sacrificio de paso de signo inverso: el abandono -también parcial- de la cultura propia, con lo cual el tránsito hacia el estatus de estudiante comporta un proceso de aculturación que implica "una serie de rupturas brutales" (Coulon, 1997, p. 37). En particular, en el proceso de afiliación intelectual cumple un papel fundamental el aprendizaje de un nuevo sistema comunicativo (Romainville, 2000; Pollet, 2000): el estudiante demuestra su adecuación a la cultura universitaria superando pruebas de comprobación de lectura bibliográfica, participando en intercambios orales formales y focalizados en saberes teóricos, exhibiendo repertorios léxicos especializados, escribiendo para comunicar lo que ha aprendido, etc. Entonces, a las numerosas rupturas con la vida anterior se le agrega la ruptura discursiva: para alcanzar la afiliación intelectual tendrán que transitar un proceso de aculturación a los discursos universitarios (Pollet, 2001).

Pero, ¿qué se espera del estudiante?, ¿qué debe hacer para aprender su métier y mantener su condición de miembro afiliado a lo largo de toda su trayectoria académica?, ¿dónde, cuándo y con quién aprende las reglas que tiene que cumplir? El estudiante encontrará las respuestas en el terreno y mediante un ejercicio permanente de desciframiento, puesto que la peculiaridad de las normas de la cultura universitaria reside en que, en su mayoría, están ocultas. Si es ingresante deberá actuar como un detective para descifrar los códigos implícitos de la nueva cultura en la que desea ser admitido (Coulon, 1995; 1997); si es estudiante avanzado tendrá que ser capaz de activar sus saberes en situaciones nuevas y prácticamente sin asistencia externa. A ambos se les exigirá autonomía, una suprarregla de la vida universitaria que a nuestro juicio adquiere diferentes significaciones según se la estudie en las dimensiones institucional o intelectual (Casco, 2009). 
Puestos a reconstruir las reglas básicas de la afiliación intelectual, consideramos que pueden distinguirse cuatro mandatos implícitos. El estudiante universitario debe: 1) valorar de manera positiva el saber legítimo y preferirlo frente a otros saberes no validados por la especulación científica; 2) utilizar categorizaciones propias del mundo intelectual; 3) movilizar sin asistencia y en nuevos contextos los conocimientos adquiridos; 4) (de)mostrar dominio del trabajo intelectual a través de producciones discursivas propias del sistema comunicativo universitario (Pollet, 2000, 2001). En relación con estos exigentes desafíos nuestro trabajo de campo nos reveló, como intentaremos mostrar en adelante, una limitada construcción del problema de la afiliación intelectual por parte de los docentes involucrados en el estudio.

\section{Metodología}

En el marco de una investigación cualitativa, las técnicas de recolección de datos que se utilizaron fueron la encuesta y la entrevista (Quivy y Campenhoudt, 2005). En una primera etapa del proceso de investigación se realizó la encuesta entre treinta profesores universitarios: diez de la Facultad de Ciencias Sociales, diez de la Facultad de Ciencias Veterinarias, y diez de la Facultad de Ingeniería de la Universidad Nacional del Centro de la Provincia de Buenos Aires, Argentina. En una segunda etapa se entrevistó a tres profesores de las mencionadas facultades. La utilización complementaria de ambos instrumentos metodológicos resultó productiva, en tanto en las entrevistas surgieron cuestiones que la encuesta no había permitido registrar.

La elección de los encuestados buscó garantizar la representatividad del universo de docentes. Por ello se administró la encuesta entre un espectro amplio de profesores de primero, segundo, tercero, cuarto y quinto años de distintas carreras pertenecientes a las tres facultades. La finalidad fue obtener respuestas susceptibles de un análisis cuantitativo general que permitiera detectar patrones o regularidades en las declaraciones de los docentes.

Una vez procesados los resultados de la encuesta, se dispuso de orientaciones para seleccionar a quienes serían entrevistados. En este caso la elección se basó en un muestreo teórico que permitió un acercamiento en profundidad a la perspectiva de los docentes a través de tres informantes calificados. Cabe señalar que el principio básico del muestreo teórico es que el número de casos retenidos no apunta a conformar una base 
propicia para la inferencia estadística, sino a seleccionar aquellos casos que suministran mayor riqueza informacional (Mucchielli, 1996).

La técnica de encuesta se aplicó mediante un cuestionario autoadministrado: los encuestados recibieron el instrumento por correo electrónico y respondieron sin orientaciones de la encuestadora. Las respuestas fueron enviadas, en su mayoría, por el mismo medio. Sólo en unos pocos casos los encuestados completaron el formulario en forma manuscrita y lo entregaron en soporte papel. Durante la administración de la encuesta, si bien se cuidó de no interferir en las respuestas, se tomaron recaudos para que la aplicación del cuestionario no presentara inconvenientes. Por ello el envío por correo electrónico contenía una breve explicación de los propósitos del estudio, así como datos básicos de la investigación en la que se enmarcaba.

Las entrevistas fueron no estructuradas o abiertas y se focalizaron en los temas, preocupaciones y observaciones relevados en las encuestas. La conversación mantenida con los entrevistados permitió no sólo ampliar los datos recogidos mediante el cuestionario sino también obtener otras informaciones relevantes para el estudio.

Finalmente es necesario aclarar que en la presentación de resultados utilizamos expresiones entrecomilladas que corresponden a extractos textuales de las respuestas recogidas en encuestas y entrevistas. Este mecanismo de recuperación literal de las palabras de los docentes es utilizado en todo el artículo.

\section{Descripción de la encuesta}

El cuestionario contenía tres partes: una introducción, un encabezamiento con espacios a completar (datos del encuestado) y seis preguntas. La introducción estaba firmada por la responsable de la investigación, indicaba el carácter anónimo y voluntario de las respuestas, y hacía explícito el propósito de recoger información sobre prácticas de comunicación académica en el nivel universitario en el marco de un estudio exploratorio sobre géneros académicos. También se aclaraba en la introducción que, aunque la encuesta era anónima, los datos académico-institucionales se solicitaban para identificar coincidencias y/o diferencias según áreas específicas de conocimiento. Los datos a completar en el encabezamiento eran los siguientes: facultad, carrera, materia, año de cursada, cargo docente, título de grado, título de posgrado, área de especialización en docencia y área de especialización en investigación. Con esas informaciones se pretendía conformar un perfil académico de los encuestados. 
La encuesta contenía tres preguntas cerradas con alternativas de respuesta que no eran mutuamente excluyentes (preguntas 1, 4 y 5). La pregunta 1 era la única en la que se solicitaba, además, jerarquizar opciones. En cuanto al resto, las preguntas 2 y 3 eran abiertas y fueron diseñadas con el propósito de que las respuestas complementaran la información obtenida en los ítems anteriores. Se esperaba que a partir de esas preguntas los sujetos encuestados reflexionaran según su experiencia docente e hicieran explícitas otras ideas que la primera pregunta cerrada no permitía consignar.

La primera pregunta buscaba que los docentes identificaran, entre una serie de opciones, problemas de los estudiantes en el desarrollo de su trayectoria universitaria. La pregunta 1 era la siguiente: Según su experiencia, ¿en cuál/es de los siguientes aspectos manifiestan mayores dificultades sus estudiantes? (Enumere por orden de importancia decreciente). A continuación se presentaba el siguiente listado: conocimientos previos sobre los objetos de estudio, curiosidad por los temas (nuevos o conocidos), estrategias de estudio, escritura, lectura comprensiva, participación en intercambios orales, resolución de problemas prácticos, seguimiento y comprensión de exposiciones del profesor, Otros (especifique).

Luego dos preguntas abiertas se dirigían a obtener información ampliatoria a través de respuestas creadas en su totalidad por los encuestados. La pregunta 2 (¿A qué atribuye usted esas dificultades?) procuraba que los docentes expusieran sus puntos de vista acerca de las posibles causas de las dificultades que habían señalado para el primer interrogante. Con la pregunta 3 (En su opinión, ¿qué acciones se deberían realizar en la Universidad para mitigar esos problemas?) se esperaba recoger datos acerca de cómo pensaban los docentes universitarios una posible intervención institucional ante los problemas de los estudiantes.

Las dos preguntas restantes, como se ha dicho, eran cerradas con alternativas de respuesta que no eran mutuamente excluyentes. La pregunta 4 solicitaba: En el siguiente cuadro marque con una cruz los textos que sus estudiantes deben producir (escribir/graficar) en situaciones de enseñanza/aprendizaje o de evaluación propias de la materia que Ud. dicta. En la primera columna del cuadro se presentaba un listado de 22 tipos de texto, entre los cuales los encuestados podían elegir varias opciones. En las dos columnas siguientes los encuestados debían especificar la situación didácticocomunicativa en la que se producían los textos (enseñanza-aprendizaje/evaluación). La pregunta 5 solicitaba marcar opciones a partir del siguiente interrogante: ¿Cuál/es de los 
siguientes procedimientos solicita que realicen sus estudiantes mediante las consignas de escritura que les presenta? En este caso se brindaba a los encuestados 26 opciones.

En cuanto a su orientación temática, en síntesis, las tres primeras preguntas aludían de manera general a la afiliación intelectual y las dos últimas se referían específicamente a la escritura. A continuación presentaremos los resultados de la encuesta teniendo en cuenta esa distinción y complementaremos nuestras observaciones con los datos obtenidos a través de las entrevistas.

\section{Resultados}

Perspectiva docente sobre problemas de afiliación intelectual

A partir de las respuestas a la primera pregunta de la encuesta puede establecerse que la percepción de los docentes acerca de las dificultades estudiantiles -restringidas las opciones, como se ha dicho, a diversos aspectos de la afiliación intelectual- se concentra en cuatro problemas: conocimientos previos, lectura, estrategias de estudio y escritura. En efecto,

- para el $33 \%$ de los encuestados la dificultad mayor se relaciona con los conocimientos previos sobre los objetos de estudio;

- para el $27 \%$ con la lectura comprensiva; y

- para el $13 \%$ con las estrategias de estudio y escritura.

Al momento de identificar el origen y/o las causas de los problemas, en las respuestas a la segunda pregunta el $60 \%$ de los encuestados responsabiliza a los propios alumnos, el $45 \%$ al nivel educativo anterior, y el $40 \%$ a factores intrínsecos de la enseñanza universitaria (la sumatoria no resulta en el $100 \%$ puesto que, al tratarse de una pregunta abierta, los encuestados señalaron más de una causa a la vez). Estos resultados muestran una tendencia que se corrobora en las entrevistas: los docentes universitarios demuestran una baja predisposición a advertir que algunas dificultades de sus estudiantes pueden producirse como consecuencia de prácticas pedagógico-didácticas propias del medio universitario.

Cabe destacar que si bien en la pregunta 1 la mayoría ha marcado un orden jerárquico entre conocimientos previos-lectura-escritura, en las respuestas a la pregunta 2 estos problemas aparecen juntos. Así, los profesores integran en un único enunciado:

- "las debilidades en lo que respecta a lectura, escritura y estrategias de estudio",

- "falta de prácticas referentes a lecto escritura [sic], comprensión de textos, etc.", 
- "falta de una correcta lecto-escritura (hay serios problemas de alfabetización, de lectura comprensiva, etc.)".

Asimismo, afirman que:

- [los estudiantes] "no poseen habilidades de comprensión lectora, no reconocen la importancia de redactar de manera clara, no releen sus propias producciones ni se detienen a 'pensar' si comprenden o no lo que leen",

- "los problemas de comprensión y de estudio se presentan de manera muy marcada en algunos alumnos",

- "los alumnos no vienen ejercitados en dedicarle horas de silla al estudio, interpretación de textos, elaboración de informes escritos, resolver situaciones problemáticas, etc. etc.”.

Los tres problemas señalados, entonces, se subsumen en uno solo. Esto podría interpretarse de manera favorable: es posible que los docentes manifiesten una percepción acertada acerca del interdinamismo entre lectura y escritura. Sin embargo también podría pensarse, en sentido contrario, que los profesores revelan en esas declaraciones una comprensión muy borrosa de las particularidades (cognitivas, didácticas, procedimentales) de los procesos involucrados.

Llama la atención también que en las respuestas a la pregunta 2, en la que se buscaba que los encuestados expusieran causas, la mayoría se limite a describir los problemas señalados en la pregunta 1. En otros casos agregan algunos problemas que no figuraban en las opciones de esa pregunta. También en las entrevistas ha ocurrido algo similar. Pareciera que a los profesores les resulta más fácil marcar dificultades de sus alumnos que generar explicaciones sobre las mismas.

En esa inclusión de nuevos problemas aparece una rica enumeración de cuestiones que corresponden, a grandes rasgos, a dos órdenes: el psicológico-actitudinal y el conceptual-cognitivo. Los ejemplos correspondientes a este segundo orden nos resultan interesantes puesto que se relacionan de manera bastante directa con cuestiones propias de la afiliación intelectual. Veamos algunos:

- "[falta de] capacidad de abstracción y de relacionar conceptos",

- "[los alumnos] ven la formación como "fragmentos' y no como un todo",

- "lo que se les enseña en el nivel anterior lo olvidan, no pueden relacionar los temas vistos, hacen borrón y cuenta nueva", 
- "no se encuentran familiarizados en [sic] la utilización del marco teórico para la interpretación de situaciones o casos",

- "[demuestran] un nivel de escolarización excesiva [sic] para (...) comprender cuestiones teóricas, abstractas, que no remitan a un ejemplo concreto".

Como puede apreciarse en los ejemplos que hemos seleccionado, los docentes refieren a deficiencias de conceptualización vinculadas con el establecimiento de relaciones e integración entre informaciones, baja activación de esquemas de conocimiento (Van Dijk, 1978, 1988), tendencia a concretizar antes que a abstraer (Castellani, 1998). Sin embargo, apenas dos de los docentes encuestados conciben la necesidad de intervenir promoviendo más prácticas de escritura en las cátedras, en especial las vinculadas con los discursos de razonamiento (Zamudio y Atorresi, 2000).

Las respuestas a la pregunta 3 también indican una escasa inclinación de los docentes a pensar soluciones que impliquen trabajo al interior de las cátedras. En efecto, al proponer acciones posibles,

- el $38 \%$ de los encuestados menciona medidas indirectas (articulación con la escuela media, modificaciones de planes de estudio, mejoras de la formación docente);

- el $33 \%$ refiere a dispositivos didácticos extra-curriculares y extra-cátedras (talleres y cursos en el período de ingreso);

- y sólo el $24 \%$ vislumbra soluciones a partir del trabajo didáctico intra-cátedras.

En lo que atañe específicamente a problemas relacionados con la escritura, sólo tres de los encuestados manifiestan conocer algo de la función epistémica (Castelló, 2005) de la misma aunque -como se ha dicho- sólo dos de ellos proponen escribir más en las cátedras.

\section{El lugar de la escritura en las prácticas didácticas}

Como se ha dicho, la pregunta 4 de la encuesta fue diseñada para relevar los tipos de textos que los estudiantes deben escribir en el marco de diferentes materias curriculares, en situaciones de enseñanza/aprendizaje o de evaluación. En la sistematización de resultados que ofrece el Cuadro 1 puede apreciarse que los porcentajes más altos corresponden a trabajos prácticos, resolución de problemas y apuntes de clase (en una franja que va del $86 \%$ al $71 \%$ de los casos). Según una descripción empírica que realizáramos en el mismo contexto institucional, esos géneros del sistema comunicativo 
universitario se caracterizan por la preeminencia de mecanismos de pregunta/respuesta, de enunciado/solución de un problema y de registro (Casco, 2010). Esto marca una tendencia que se corrobora en las entrevistas: el predominio de actividades de escritura comprobatoria y reproductiva.

En un segundo grupo de respuestas, que ocupa una franja que va del $43 \%$ al $29 \%$ de los casos, aparece la opción por nueve tipos textuales más, entre los cuales sólo tres permitirían vislumbrar mecanismos discursivos generadores de mayores desafíos cognitivos para los estudiantes (comentario de texto, informe, revisión bibliográfica). E1 resto de las opciones, constituido por tipos textuales propios del desarrollo completo de ideas, obtiene porcentajes comparativamente muy bajos.

Cabe señalar que no existe correspondencia entre los tipos discursivos solicitados en instancias de enseñanza-aprendizaje y los requeridos con fines evaluativos. A los estudiantes se les pide escribir para cumplir con los requisitos de las materias curriculares pero el saber-hacer implicado se da por sentado. Se espera que los alumnos respondan "espontáneamente" a cualquier consigna de escritura, sea ésta para enseñar o para evaluar. Interpretamos tal incongruencia como una prueba de la invisibilidad de las prácticas de escritura en la universidad y el escaso conocimiento que tienen los profesores sobre la complejidad de los procesos involucrados.

Cuadro 1. Textos que deben producir los estudiantes en las diferentes materias curriculares

\begin{tabular}{|l|c|c|}
\hline \multirow{2}{*}{\multicolumn{1}{c|}{ Textos }} & $\begin{array}{c}\text { Enseñanza- } \\
\text { aprendizaje }\end{array}$ & Evaluación \\
\cline { 2 - 3 } & $(\mathrm{en} \%)$ & (en \%) \\
\hline Trabajo práctico & 86 & 24 \\
\hline Resolución de problema & 76 & 57 \\
\hline Apunte de clase & 71 & 0 \\
\hline Comentario de texto & 43 & 19 \\
\hline Gráfico & 43 & 38 \\
\hline Informe & 43 & 24 \\
\hline Cuadro & 38 & 19 \\
\hline Respuesta a cuestionario de preguntas abiertas & 38 & 48 \\
\hline Revisión bibliográfica & 38 & 14 \\
\hline Examen final & 33 & 57 \\
\hline Examen parcial & 29 & 62 \\
\hline Respuesta a guía de lectura & 29 & 10 \\
\hline Ensayo & 24 & 10 \\
\hline Fichaje & 24 & 0 \\
\hline Mapa o red conceptual & 24 & 0 \\
\hline Reseña & 14 & 5 \\
\hline Resumen & 14 & 5 \\
\hline Trabajo final & 14 & 24 \\
\hline Monografía & 10 & 10 \\
\hline
\end{tabular}




\begin{tabular}{|l|c|c|} 
Proyecto de investigación & 10 & 0 \\
\hline Respuesta a cuestionario multiple choice & 0 & 19 \\
\hline Respuesta a un test & 0 & 10 \\
\hline Otros (especifique) & 0 & 0 \\
\hline
\end{tabular}

En el Cuadro 2 se presenta la sistematización de las respuestas a la pregunta 5. Mediante este último ítem buscábamos que los encuestados identificaran los procedimientos contenidos en las consignas de escritura. La palabra procedimientos que aparece en la pregunta no corresponde estrictamente a terminología científica y fue usada con un sentido general que permitiera hacer más comprensible la interrogación. Sin embargo, las opciones presentadas sí fueron pensadas sobre la base de los supuestos teóricos establecidos por Jorba (2000), quien propone un modelo recursivo en el que interactúan habilidades cognitivas, habilidades cognitivolingüísticas y tipos de textos. Según este autor, existen habilidades que están en la base de operaciones cognitivas que se producen constantemente en las actividades de aprendizaje y de estudio. Esas habilidades cognitivas -analizar, comparar, clasificar, etc.- "reclaman" habilidades cognitivolingüisticas que se activan al producir un texto -explicar, argumentar, definir, resumir, etc.- $\mathrm{y}$, de hecho, se relacionan directamente con las tipologías textuales (Jorba, 2000).

Ahora bien, de acuerdo con las declaraciones de los docentes, las consignas de escritura obligarían a desplegar habilidades cognitivas y habilidades cognitivolingüísticas de alto nivel, es decir, aquellas que suponen un gran esfuerzo intelectual. En efecto, entre las habilidades cognitivas más estimuladas están: analizar (76\%), interpretar (71\%), comparar (67\%), clasificar (57\%); mientras entre las habilidades cognitivolingüísticas se registran: fundamentar (90\%), explicar (67\%), argumentar (62\%), definir (57\%). Estos datos resultan paradójicos, si se tiene en cuenta que rara vez esos procedimientos son objeto de enseñanza en la universidad argentina (Carlino, 2005) y conocemos que sólo en contadas excepciones lo son en la institución en la que se realizó el estudio. De modo que las manifestaciones de los docentes revelarían la expectativa de que los estudiantes realicen "naturalmente" las operaciones referidas. 
Cuadro 2. Procedimientos discursivos de las consignas de escritura

\begin{tabular}{|l|c|}
\hline \multicolumn{1}{|c|}{ Procedimientos } & en \% \\
\hline Fundamentar & 90 \\
\hline Analizar & 76 \\
\hline Interpretar & 71 \\
\hline Explicar & 67 \\
\hline Ejemplificar & 67 \\
\hline Comparar & 67 \\
\hline Argumentar & 62 \\
\hline Esquematizar & 57 \\
\hline Definir & 57 \\
\hline Clasificar & 57 \\
\hline Graficar & 52 \\
\hline Diferenciar & 52 \\
\hline Sintetizar & 48 \\
\hline Relacionar & 48 \\
\hline Opinar & 48 \\
\hline Evaluar & 33 \\
\hline Comentar & 33 \\
\hline Categorizar & 33 \\
\hline Caracterizar & 33 \\
\hline Sistematizar & 29 \\
\hline Resumir & 29 \\
\hline Reformular & 24 \\
\hline Generalizar & 24 \\
\hline Contraargumentar & 0 \\
\hline Recodificar & \\
\hline Expandir & 19 \\
\hline & 29 \\
\hline & 58 \\
\hline
\end{tabular}

Por su parte las entrevistas otorgan una evidencia importante acerca del lugar que tiene la escritura en las aulas universitarias: la proporción de obligaciones de lectura para el estudiante es mucho mayor que la cantidad de escrituras que tiene que producir para cumplir con los requisitos académicos. Además, el tipo, cantidad y calidad de textos objeto de lectura es mucho mayor que el tipo, cantidad y calidad de escritos que se deben producir a lo largo de los años de formación. De modo que la escritura es una práctica poco ejercitada en relación con la lectura.

Otra evidencia que proporcionan las entrevistas tiene que ver precisamente con la riqueza y variedad de las escrituras exigidas a los estudiantes. Si bien frente a la pregunta 4 de la encuesta los docentes marcan una considerable cantidad de tipos discursivos solicitados con fines académicos, las entrevistas permitieron establecer que, en definitiva, la mayor parte se resuelve con base en mecanismos recurrentes de pregunta/respuesta (para todas las carreras), de enunciado/solución de un problema (fundamentalmente para la carrera de Ingeniería, pero también para Ciencias 
Veterinarias) y de exposición escrita monológica por parte del alumno (para todas las carreras). Estas observaciones permiten afirmar que las producciones discursivas en situaciones de enseñanza-aprendizaje con frecuencia se cristalizan en formatos de escritura reproductiva (Rosales y Vázquez, 2008) en los que prima el fragmentarismo, la descontextualización y la "extracción de contenido" desde las fuentes objeto de lectura (o desde la fuente oral que constituye el profesor).

Es verdad que -en proporción comparativamente muy baja- algunos profesores también ponen a sus estudiantes frente a tareas de escritura más complejas y que implican desafíos retóricos y cognitivos de mayor profundidad. Pero de las entrevistas se desprende que los aprendices se adecuan a estos requerimientos "al tanteo", puesto que no se destina tiempo didáctico a proporcionar modelos, construir con los estudiantes definiciones situadas de los tipos discursivos involucrados, reflexionar sobre la forma en que se van resolviendo problemas retóricos, hacer devoluciones o retroalimentación.

Cabe problematizar, además, la distancia existente entre los formatos de escritura reproductiva producidos en situaciones de enseñanza-aprendizaje y los escritos propios de cada área de saber disciplinar. Sabemos, en principio, que pertenecen a dos esferas bien delimitadas de la actividad humana: los primeros se producen en una comunidad discursiva de aprendizaje y los segundos en una comunidad discursiva profesional o científica. Sin embargo, es de esperar que haya lazos entre esas dos esferas, algo que es muy difícil de establecer en el caso estudiado. En los procesos de formación en los espacios áulicos de la primera esfera se reconstruyen saberes mediante procesos de acercamiento (ficcionalización) al contexto de la segunda (Milian, 2007). De hecho, para algunos autores la misión pedagógica fundamental sería conducir a quienes se educa hacia la adquisición progresiva del poder de los lenguajes especializados (Bazerman, 1988 y Kress, 1985 y 1993, citados por Milian, 2007). El trabajo docente, por tanto, debería propender a estimular el establecimiento de relaciones entre la comunidad de aprendizaje y la comunidad científica de referencia. En nuestro caso es muy borrosa la vinculación entre ambas, lo cual constituye un dato llamativo, aunque se necesitaría un estudio mucho más profundo para hacer afirmaciones seguras al respecto.

El panorama que hemos explorado nos indica que en nuestras aulas universitarias se brindan pocas oportunidades de estimular operaciones cognitivas por vía de la producción escrita. Sin duda esta es una condición que obstaculiza las 
posibilidades de afiliación intelectual de los alumnos. Langer y Applebee (1987, citados por Milian, 2007) señalan que la escritura promueve el aprendizaje en grado mayor que las actividades de estudio o de lectura; que diferentes actividades de escritura conducen a distintos tipos de información; que la escritura analítica estimula el trabajo intelectual de búsqueda y descubrimiento. Esos potenciales aportes de la escritura al desarrollo intelectual no parecen ser aprovechados en nuestra institución: se escribe mucho menos de lo que se lee; se realizan actividades de escritura poco variadas; se obstruye la escritura analítica con escritos centrados en la búsqueda de respuestas breves o en la resolución de ejercicios puntuales.

El proceso de composición (Flower y Hayes, 1981; Bereiter y Scardamalia, 1987) involucra intensa actividad cognitiva y es poco visible desde el exterior, por eso constituye un gran desafío para quien enseña. Se necesita un docente que conozca bien ese proceso y administre parte de sus horas de clase para estimularlo (Castelló y Milian, 1997, p. 118). El poder epistémico de la escritura se experimenta cuando, frente al desafío retórico de responder mediante un escrito a una necesidad auténtica de la comunicación social, nos vemos obligados a volver una y otra vez sobre las ideas que vamos construyendo, la representación del texto a escribir, su audiencia, etc. En suma, la escritura reflexiva necesita tanto una guía externa como tiempo para desplegarse. Pero las condiciones propias del medio universitario, y dentro de ellas de manera central la disponibilidad de tiempo didáctico, hacen muy dificil pensar en prácticas de escritura a través del curriculum (Bazerman, et al., 2005).

\section{Actitudes, supuestos y creencias de los docentes}

Los docentes universitarios entrevistados tienden a responsabilizar al nivel educativo anterior y/o los propios alumnos por las dificultades discursivas y de aprendizaje. Esta tendencia, ya reconocida en los resultados de la encuesta, se profundiza en las entrevistas. En su mayoría los profesores razonan como si la escuela media tuviera que entregar estudiantes 'listos para usar', es decir, sujetos ya preparados para enfrentar todas las exigencias de la afiliación intelectual.

Esta posición del profesorado se corresponde con las decisiones institucionales de las últimas décadas. Ante los problemas de lectura, escritura y estrategias de trabajo intelectual, las distintas facultades han ido incorporando dispositivos extracurriculares que se presentan en términos remediales (de 'compensación' o de 'nivelación' de 
conocimientos). Se suscribe así la idea de que 'el tratamiento' para las dificultades estudiantiles -valga la metáfora médica- implica una intervención a corto plazo y no requiere trabajo didáctico en las áreas curriculares de la formación específica. De hecho, cuando los profesores son impulsados a pensar en soluciones, casi siempre las conciben como intervenciones por fuera de las estructuras curriculares. La mayoría habla de espacios institucionales a cargo de especialistas en didáctica de la lengua o disciplinas afines, ubicados en períodos iniciales o paralelos al dictado de las materias curriculares. No parece haber todavía predisposición para admitir la necesidad de construcciones didácticas específicas para el nivel superior.

En lo que refiere a las prácticas comunicativas propias del proceso de afiliación intelectual, las respuestas de los encuestados y entrevistados permiten entrever la vigencia de supuestos erróneos. En primer lugar, en sus declaraciones campea una concepción inexacta sobre el aprendizaje de la lectura y la escritura. Tal concepción puede resumirse así: el individuo aprendería a comunicarse oralmente y por escrito durante un período limitado (escolaridad básica) y, una vez realizado ese aprendizaje acabado y definitivo-, estaría en condiciones de pasar a la etapa de incorporación al mundo de los saberes legítimos (es decir, legitimados por las instituciones científicas). Al respecto es sugestivo que varios docentes utilicen los términos alfabetización o lecto-escritura para referirse a la 'falta' que observan en sus estudiantes. De este modo manifiestan el preconcepto de que la escolaridad obligatoria estaría destinada a asegurar lectores y escritores plenos y expresan la frustración ante estudiantes universitarios, si no analfabetos, al menos iletrados (Ferreiro, 2003). Ignoran, así, la idea de alfabetización con sentido amplio, es decir, como medio para el acceso universal a la cultura escrita (Torres, 2006).

En segundo lugar, y en relación directa con lo antedicho, también aparece en los testimonios de los docentes la suposición de que el éxito en los estudios universitarios dependería del dominio de ciertas preadquisiciones intelectuales generales: capacidades de abstracción, de lectura comprensiva, de escritura, de técnicas de estudio, de resolución de problemas. Se trata de una visión bastante generalizada que, tal como nos señala Romainville (2000) para el contexto universitario francófono, no tiene real poder explicativo ni sustento en las investigaciones vigentes.

En tercer lugar, las declaraciones de los encuestados y entrevistados revelan la creencia en la separación pensamiento-lenguaje. En efecto, estos profesores afirman con 
frecuencia que sus estudiantes poseen el conocimiento pero no saben expresarlo debido a sus carencias lingüísticas. Este tipo de afirmaciones nos reenvía al supuesto de que habría capacidades generales que se pueden adquirir en los niveles educativos anteriores $\mathrm{y}$ hacer funcionar en otros contextos con independencia de los procesos cognitivos involucrados.

\section{Conclusiones}

La concepción del ingreso como un arduo pasaje a otra cultura se opone al presupuesto de la progresión automática de la escuela media a la universidad. Al respecto la metáfora oficio de estudiante, como el propio Coulon lo ha afirmado, resalta el carácter no natural ni espontáneo del proceso (Becquet, 1999). Y tanto él como los autores que han recogido su concepto de afiliación demuestran que se trata de un proceso no armónico sino conflictivo para el sujeto, en razón de su necesidad de romper parcialmente con la cultura de la que proviene. Además, la diferenciación entre afiliación institucional y afiliación intelectual da cuenta de la complejidad del proceso y otorga sentido al reclamo por una iniciación sistemática a las normas de la cultura universitaria: Coulon (1997) promueve una pedagogía de la afiliación, Romainville (2004) una didáctica universitaria dirigida a desmontar el implícito universitario, Pollet (2001) una didáctica de los discursos universitarios.

Con base en este enfoque teórico nos hemos interrogado por la perspectiva que ha construido y manifiesta el profesorado en torno al fracaso de los estudiantes universitarios. Nuestro interés principal era reconstruir -al menos parcialmente- su visión de la afiliación intelectual, con especial atención a las expectativas, supuestos y prácticas en torno a la escritura. Y ello debido a que en el proceso de afiliación intelectual cumple un papel fundamental el aprendizaje del sistema comunicativo universitario.

Los resultados, como se ha podido apreciar, nos muestran un terreno poco fértil para una pedagogía de la afiliación. Los docentes tienden a cargar sobre los propios estudiantes toda la responsabilidad por sus fracasos y no parecen vislumbrar la posibilidad de mitigarlos con una intervención desarrollada en el propio medio universitario. A nuestro modo de ver, esta actitud de los profesores no se deriva de una postura personal negligente o indiferente (de hecho, el conjunto de nuestros encuestados y entrevistados aceptó participar de manera voluntaria en el trabajo de campo y 
manifestó una fuerte preocupación por el tema) sino de la vigencia de presupuestos erróneos y condiciones institucionales poco propicias.

Según la perspectiva de los docentes, cuatro son los aspectos en que los estudiantes manifiestan mayores dificultades: conocimientos previos sobre los objetos de estudio, lectura comprensiva, estrategias de estudio y escritura. En general los profesores demuestran poco conocimiento y comprensión sobre el origen de esos problemas. Algunos intentan describirlos mediante la provisión de ejemplos y anécdotas, pero no logran generar mayores explicaciones sobre los mismos. Y aquellos que, aunque de manera borrosa, tratan de explicar las dificultades de sus alumnos, sobrevaloran las preadquisiciones. Esta última postura, como indica Romainville (2000), conduce al fatalismo y permite a los docentes desentenderse de los fracasos.

Finalmente, es sugestiva la homogeneidad de declaraciones relativas a las prácticas de comunicación que hallamos tanto en encuestas como en entrevistas. Los profesores de la Facultad de Ciencias Sociales hacen afirmaciones similares a las de los profesores de Ingeniería o de Ciencias Veterinarias, hecho que nos lleva a sospechar que la comunidad discursiva de aprendizaje funciona con una dinámica excesivamente independiente de las especificidades propias de cada área del saber científico. Los formatos escolarizados de pregunta-respuesta o de resolución de problemas reinan por igual en las tres carreras y atraviesan las prácticas de escritura de primero a quinto año, de modo que el acercamiento de los estudiantes a los géneros de la esfera profesional o científica se restringe sólo a las prácticas de lectura. Los mismos profesores señalan que recién al final de la carrera los estudiantes deben enfrentarse con un desafío de escritura complejo y vinculado de manera más directa a la práctica profesional (tesis, en Ciencias Sociales; tesina, en Ciencias Veterinarias; trabajo de integración final, en Ingeniería), lo que suele retrasar su graduación por varios años. En definitiva, para nosotros queda abierto el problema de los géneros discursivos en función de la comunidad científica y/o profesional en la que se inscriben, puesto que sospechamos que los estudiantes permanecen por mucho tiempo - a veces hasta el final de sus carreras- en la sala de espera de los universos disciplinares de sentido. 


\section{Referencias bibliográficas}

Aguilar Rivera, M. (2007). La transición a la vida universitaria. Éxito, fracaso, cambio $y$ abandono. Ponencia presentada en el IV Encuentro Nacional de Docentes Universitarios Católicos (ENDUC IV), Santa Fe, Argentina.

Araujo, S. (2008). Características del proyecto de investigación. En Formación universitaria y éxito académico: Disciplinas, estudiantes y profesores (pp. 1748). Tandil, Argentina: Editorial de la Universidad Nacional del Centro de la Provincia de Buenos Aires.

Bazerman, C. (1988). Shaping written knowledge: The genre and activity of the experimental article in science. Madison, WI: University of Wisconsin Press.

Bazerman, C., Little, J., Bethel, L., Chavkin, T., Fouquette, D. y Garufis, J. (2005). Reference guide to writing across the curriculum. Indiana: Parlor Press/WAC Clearinghouse.

Becquet, V. (1999). L'Art d'être étudiant. Un entretien avec Alain Coulon, chercheur en sciences de l'éducation. Factuel: La Revue, 3, 33-36.

Bereiter, C., y Scardamalia, M. (1987). The psychology of written composition. Hillsdale, N.J: Erlbaum.

Carlino, P. (2005). Escribir, leer, y aprender en la universidad. Una introducción a la alfabetización académica. Buenos Aires: Fondo de Cultura Económica.

Carlino, P. (2007). ¿Qué nos dicen las investigaciones internacionales sobre escritura en la universidad? [versión electrónica]. Cuadernos de psicopedagogía. 4, 21-40.

Casco, M. (2009). Afiliación intelectual y prácticas comunicativas de los ingresantes a la universidad. Co-herencia, 6(11), 233-260.

Casco, M. (2010). Ocurrencias discursivas del sistema comunicativo universitario en dos áreas de la formación superior: Ingeniería y Ciencias Sociales (Universidad Nacional del Centro de la Provincia de Buenos Aires). Barcelona, Cataluña: Facultad de Ciencias de la Educación, Universidad Autónoma de Barcelona, UAB. Trabajo final de Máster Universitario en Investigación en Didáctica de la Lengua y la Literatura.

Castellani, D. (1998). La manifestación de problemas conceptuales en los textos de estudiantes universitarios. Intersecciones. Revista de la Facultad de Ciencias Sociales, Universidad Nacional del Centro de la Provincia de Buenos Aires. II (2/otoño), 31-52.

Castelló, M. (2005). La escritura epistémica: Concepciones y estrategias de regulación en estudiantes de doctorado. Ponencia presentada en la IV Jornada de Desarrollo Humano y Comunicación Alcalá de Henares, Madrid.

Castelló, M. y Milian, M. (1997). Enseñar y aprender estrategias en el proceso de composición escrita. En Pérez Cabaní, A.M.L. (coord.). La enseñanza y el aprendizaje de estrategias desde el curriculum (pp. 117-136). Girona: UdG/Horsori.

Coulon, A. (1995). Etnometodología y educación. Barcelona: Paidós.

Coulon, A. (1997). Le métier d'étudiant. L'entrée dans la vie universitaire. Paris: Presses Universitaires de France.

Ferreiro, E. (2003). Pasado y presente de los verbos leer y escribir. Buenos Aires: Fondo de Cultura Económica.

Flower, L., y Hayes, J. (1981). A cognitive process theory of writing. College Composition and Communication, 365-387. 
Guzmán Gómez, C. (2002). Reflexiones en torno a la condición estudiantil en los noventa: los aportes de la sociología francesa. Perfiles Educativos, XXIV(9798), 38-56.

Jorba, J. (2000). La comunicación y las habilidades cognitivolingüísticas. En J. Jorba, I. Gómez, y A. Prat (Coords.). Hablar y escribir para aprender. Uso de la lengua en situación de enseñanza-aprendizaje desde las áreas curriculares (pp. 29-49). Madrid, España: Editorial Síntesis.

Kress, G. (1985). Linguistic processes in sociocultural practice. Oxford: Oxford University Press.

Kress, G. (1993). Genre as social approach. En B. Cope y M. Kalantzis (coords.) The Powers of Literacy (pp. 22-37). London: The Falmer Press.

Langer, J., \& Applebee, A. (1987). How writing shapes thinking: A study of teaching and learning. Urbana, IL: NCTE.

Milian, M. (2007). Escribir a través del curriculum: el lenguaje como instrumento transversal para el aprendizaje. Barcelona: Departamento Didàctica de la Llengua i la Literatura, UAB.

Mucchielli, A. (1996). Dictionnaire des méthodes qualitatives en sciences humaines et sociales. Paris : Armand Colin.

Pollet, M. Ch. (2000). Les étudiants face aux discours universitaires: de la réception d'un savoir stabilisé à celle d'un savoir en construction. Pratiques de l'écrit et modes d'accès dans l'enseignement supérieur (2), Ateliers, Cahiers de la Maison de la Recherche Université Ch. de Gaulle-Lille, 3(25), 11-25.

Pollet, M. Ch. (2001). Pour une didactique des discours universitaires. Étudiants et système de communication à l'université. Bruxelles: De Boek Supérieur.

Quivy, R., y Campenhoudt, L. (2005). Manual de investigación en Ciencias Sociales. México: Noriega Editores.

Romainville, M. (2000). L'échec dans l'université de masse. Paris: L'Harmattan.

Romainville, M. (2004). Esquisse d'une didactique universitaire. Revue francophone de gestion, 5-24.

Rosales, P., y Vázquez, A. (2008). La escritura de textos argumentativos a partir de fuentes contrastantes. Lectura y escritura críticas: perspectivas múltiples. Cátedra UNESCO, subsede Tucumán, Argentina, Recuperado el 18 de enero de 2009, de www.filo.unt.edu.ar/jorn_unesco/jorn_unesco_cd.htm.

Torres, M. (2006). Alfabetización y aprendizaje a lo largo de toda la vida. Revista Interamericana de Educación de Adultos, 28(1), 25-38.

Van Dijk, T. (1978). La ciencia del texto. Buenos Aires: Paidós.

Van Dijk, T. (1988). Estructuras y funciones del discurso. Una introducción interdisciplinaria a la lingüistica del texto y a los estudios del discurso. México: Siglo XXI Editores.

Zamudio, B., y Atorresi, A. (2000). La explicación. Buenos Aires: Eudeba.

\footnotetext{
Referencia de la autora:

Miriam Casco es Profesora de Castellano y Literatura por el Instituto Superior de Profesorado "San José" (Tandil, Buenos Aires), Magister en Lingüística Hispánica por el Instituto Caro y Cuervo de Colombia y Máster Universitario en Didáctica de la Lengua y la Literatura por la Universidad Autónoma de Barcelona. Desarrolla tareas de docencia, investigación y gestión académica en la Universidad Nacional del Centro de la Provincia de Buenos Aires. En esta institución es Directora del Departamento de Lenguas (Rectorado) y Profesora Adjunta del Taller de Producción de Textos (Facultad de Ciencias Sociales). En su trayectoria docente se destaca la coordinación de numerosos talleres de escritura destinados a estudiantes y docentes de grado y de posgrado. Como investigadora se interesa por la
} 
incidencia de las prácticas de lectura y escritura en los procesos de ingreso y permanencia en la universidad.

\section{Email: miriamcasco@speedy.com.ar}

${ }^{\mathrm{i}}$ La investigación se llevó a cabo en el año 2009 para el seminario Investigación en Didáctica de la Lengua Escrita, coordinado por la profesora Marta Milian Gubern, en el marco del Máster Universitario en Investigación en Didáctica de la Lengua y la Literatura, Facultad de Ciencias de la Educación, Universidad Autónoma de Barcelona.

Para citar este artículo:

Casco, M. (2014). Afiliación intelectual y escritura en la universidad: Un estudio de caso sobre la perspectiva de los docentes. Bellaterra Journal of Teaching \& Learning Language \& Literature, 7(2), 3453. 